\title{
Epidemiological study of pregnant women admitted to the emergency department
}

\section{Seyedhossein Ojaghihaghighi', Samad Shams Vahdati ${ }^{2}$, Simin Taghavi ${ }^{3}$, Aydin Rahimzade Jahandari', Pegah Sepehri Majd ${ }^{1}$, Mohammad Mirza-Aghazadeh-Attari ${ }^{4}$}

\author{
'Emergency Medicine Research Team,Tabriz University of Medical Sciences, Tabriz, Iran \\ ${ }^{2}$ Road Traffic Injury Research Center, Tabriz University of Medical Sciences, Tabriz, Iran \\ ${ }^{3}$ Department of Gynecology, Tabriz University of Medical Sciences, Tabriz, Iran \\ ${ }^{4}$ Medical Philosophy and History Research Center, Tabriz University of Medical Sciences, Tabriz, Iran
}

\begin{abstract}
Received: 25 February 2017
Accepted: 23 May 2017

Published online: 31 May 2017

*Corresponding author: Pegah Sepehri Majd, Ghoghasht Street, Emergency Department, Imam Reza Hospital, Tabriz, Iran.

Tel: +98-4133352078

Email: pegah.sm87@gmail.com

Competing interests: None.

Funding information: None.

Citation: Ojaghihaghighi S, Vahdat SS, Taghavi S, Rahimzade Jahandari A, Sepehri Majd P, Mirza-AghazadehAttari M. Epidemiological study of pregnant women admitted to the emergency department. Journal of Emergency Practice and Trauma 2018; 4(1): 44-47. doi: 10.15171/ jept.2017.21
\end{abstract}

\begin{abstract}
Objective: Pregnancy is an important psychological and biological phenomenon in women's life. Pregnancy has many complications jeopardizing the well-being of the mother and the child.

Methods: In this retrospective study, the data including demographic information, chief complaint, the initial diagnosis, referral decision, final diagnosis, hospitalization outcome and pregnancy outcomes were studied on 239 pregnant women admitted to the emergency departments of the general hospitals of East Azerbaijan province.

Results: The average age of patients was 27.54 years. The time period from the initial presentation to completely leave the emergency department was reported to be 3.66 hours on average. In this study, most patients $(74.5 \%)$ did not have a history of abortion. Exploring the time of admission for all cases, most patients were admitted from 8 PM to 8 AM $(67.8 \%)$, and $32.2 \%$ were referred from 8 AM to 8 PM. Concerning the time of pregnancy, $46.4 \%$ were at their second trimester of pregnancy. Most of the patients were admitted in cold seasons of the year. The most common chief compliant was abdominal pain with $32.2 \%$ frequency. In addition, the most frequent primary diagnoses were an acute abdomen and trauma at $25.5 \%$ and $14.2 \%$ respectively.

Conclusion: Abdominal pain and acute abdomen have the most frequency as the chief compliant and initial diagnosis especially during the second trimester of pregnancy. In this regard, appropriate training and skillful staff are needed to deal with the complaints and complications of abdominal pain. More than half of pregnant women referred to the emergency departments were hospitalized or admitted to the intensive care unit (ICU). This signals the necessity of adequate facilities to provide proper care for this group. Keywords: Pregnant women, Emergency service, General hospitals
\end{abstract}

\section{Introduction}

The phenomenon of pregnancy for every woman is a beautiful and pleasant experience which is associated with many physiological changes. The maximum physiological stress during a woman's life is the initiation of pregnancy. This causes the most important changes in their lives (1). In addition to some minor problems such as back pain, morning nausea and constipation, women endure many changes during their pregnancy. But in some cases, the pregnant woman has some diseases or illnesses during the pregnancy (2). Some of these problems only affect the mother, but some of them affect the mother and the fetus. Such a condition may be hazardous to the health of the mother and the fetus during pregnancy. Therefore, prenatal care is essential and important to be conducted in many aspects (3), with unintended pregnancy having many social and economic consequences. The most common diseases that lead to the death of pregnant mothers are coexisting diseases such as heart disease, diabetes, high blood pressure, rheumatic diseases and renal failure (3). Bleeding, gestational hypertension and heart diseases are the most important factors influencing maternal mortality $(4,5)$. It is of great importance to take these factors into account regarding maternal health and pregnancy related diseases as they pose a great burden on the health system financially and negatively affect families in many ways (6). The present study was conducted to evaluate the pregnant women referred to emergency departments of general educational hospitals of East Azerbaijan province from 2013 to 2014 . Identifying the 
main complaints, diagnosing the disease with nonmidwifery causes, demographic factors, and treatment are all important issues in this regard. To improve the quality and quantity of services and care that is necessary for this group of patients, it is essential to prepare medical centers for life-threatening conditions. In this regard, the public health will be promoted.

\section{Methods}

In this retrospective study, the records of all pregnant women admitted to general hospitals of East Azerbaijan province were studied and the required information obtained. These included demographic data (patient age, gestational age, number of pregnancies, abortion), chief complaint, the initial diagnosis, decisions taken in the emergency departments (discharge, hospitalized, admitted to intensive care unit (ICU), dispatched from the center or death), the final diagnosis, hospitalization outcomes (complete remission, partial remission or death and disease control) and pregnancy outcomes (one of the cases of premature birth, abortion or fetal death or continue the pregnancy). The inclusion criteria encompassed all pregnant women admitted to general hospitals of East Azerbaijan. Exclusion criteria included patients with a legal debate regarding their treatment or diagnosis at the time of the study and patients with contradicting information in their medical records.

In order to gather data, we developed a questionnaire. The validity and reliability of the questionnaire was approved by an expert panel in the deputy of research in Tabriz University of Medical Sciences. Before completing the questionnaire, the aim and purpose of the study were explained to data extractors.

Statistical analysis was done by SPSS version 16.0.0 IBM ${ }^{\bullet}$. Paired sample $t$ test was used for comparing the means. For qualitative variables, chi-square or Fisher exact test was used. The $P$ value less than 0.05 was regarded as statistically significant.

\section{Results}

Totally, 239 pregnant patients were enrolled into this study. All of them were examined during 2013 and 2014. The mean age of patients was 27.54 (16-38), and most of the patients were between 20-35 years. The mean time of stay at gynecology ward was 3.66 hours (0.5-8). $74.5 \%$ of patients had no history of a previous abortion. $54.8 \%$ had no history of delivery, $35.6 \%$ had a history of only one delivery, $8.8 \%$ had $2,0.4 \%$ had 3 and $0.4 \%$ had 4 times delivery. Concerning the number of pregnancies, $39.3 \%$ of patients were experiencing their first pregnancy, $41.0 \%$ the second, $16.7 \%$ the third, $1.7 \%$ the fourth, $0.4 \%$ the fifth and $0.8 \%$ the seventh pregnancy. Most of the patients were admitted between 8 AM up to 8 PM (67.8\%).28.9\% of patients were in their first trimester and $46.4 \%$ and $24.7 \%$ were in second and third trimester respectively. Most patients were admitted in winter (31.4\%), 25.52\% in fall, $24.69 \%$ in summer and $18.41 \%$ in spring. This is due to the high frequency of respiratory disorders in colder weather. Some of the most common chief complaints included abdominal pain (32.2\%), discomfort after trauma to the abdomen (10.9\%), cough and dyspnea (8.8\%), headache and dizziness (7.1\%), burns (6.3\%), flank pain (5\%), hematemesis $(3.8 \%)$, falling from a height $(1.7 \%)$, poisoning (1.7\%), symptoms of poor glycemic control (1.7\%), and others (20.9\%). The most common primary diagnosis was acute abdomen with a frequency of $25.5 \%$. Of the total sample, $39.3 \%$ were admitted to hospital, $13.4 \%$ to ICU, $4.2 \%$ were referred (transferred), $42.7 \%$ were discharged and $0.4 \%$ of cases were expired. Totally, $2.9 \%$ of patients expired. Noteworthy, admission in ICU was higher in patients having cough and dyspnea. This is due to the fact that these symptoms are the grim predictor of pulmonary embolism, which happen in pregnant women because of their predisposition for venous thromboembolism (7). Pregnancy continued in 92\% of patients, $4.2 \%$ had an abortion and $3.8 \%$ had preterm labor. Based on our findings, 8 deaths occurred. These included one case of sepsis regarding intestinal gangrene, one case of sepsis and DIC (disseminated intravascular coagulation), one case of DIC regarding the HELLP syndrome, one case of septic shock followed by metastatic choriocarcinoma, one case with pregnancy fatty liver (hepatic encephalopathy), one case with $60 \%$ burns, one case with respiratory arrest followed by tonic seizures, and one case complicated with ARDS (acute respiratory distress syndrome) regarding viral pneumonia. No significant relationship was found between the gestational age and the initial complaint $(P=0.78)$. There was a significant relationship between the initial complaint and decision to admit the patient to ICU $(P=0.001)$. The highest proportion of ICU admission was respectively related to "cough and shortness of breath" and "Burn". We did not find a significant relationship between the initial complaint and the season of the initial presentation $(P=0.792)$. There was a significant relationship between the primary complaint and hours of admission $(P=0.006)$. All of the cases had complaints except trauma and burns. The most common time referred was 8 AM to 8 PM. concerning burns, most patients came between 8 PM to 8 $\mathrm{AM}$, and in trauma to the abdomen the admission rate was equal in the two time periods. There was no significant relationship between the final diagnosis and gestational age $(P=0.354)$. In addition, no significant relationship was observed between the ward the patients were referred to and gestational age $(P=0.055)$.

\section{Discussion}

In the present study, data including demographic characteristics, chief complaint, the initial diagnosis, referral decision, final diagnosis, hospitalization outcome and pregnancy outcomes were studied on 239 pregnant women admitted to the emergency departments of the general hospitals of East Azerbaijan province. Based on the findings, the most frequent primary diagnoses were acute abdominal and trauma with a frequency of $25.5 \%$ and $14.2 \%$ respectively. Early diagnosis of acute abdomen 
was abdominal pain. Abdominal pain had the most frequently among initial complaints, and acute abdomen had the highest frequency among the primary diagnoses. In $33.1 \%$ of cases, the initial diagnosis did not match the final diagnosis, showing the importance of the health of pregnant women. Because of restriction on use of drugs and other therapeutic interventions during pregnancy, cause of the low percentage of complete remission and high share of partial remission with disease. Patel et al assessed the referral rates of pregnant women in the Indian state of Gujarat. They found that the most common reasons for referral to tertiary and secondary medical centers consisted of non-progressive labor (14.8\%), severe anemia (10.3\%), pre-eclampsia (10.3\%), mal-presentation (9.7\%)and postpartum hemorrhage $(9.7 \%)$ (8). In our study most of the referrals were due to abdominal pain and trauma. Complications due to anemia are not a major problem for the Iranian health system because of intensive nutritional supplementation for women starting early in adulthood (9). Ndidi and Oseremen conducted a study among pregnant women receiving antenatal care and found that $73.4 \%$ of women receiving care were in their second trimester and $26.4 \%$ in their third. This indicates that only a minority of women needed medical attention in their first trimester (10). This is in line with our results in which most patients were in the second trimester. This is reversed in many countries with extensive public health measures in which screenings and routine physical examinations start in the first trimester. Solanke et al studied the liver profile in pregnant women in western India and found that most diseases related to the hepatobiliary system were presented in the third trimester and the most common etiologic factor was viral hepatitis especially hepatitis E (11). In our study, the only cause of death because of hepatobiliary etiologies was due to fatty liver disease and no case of hepatitis was observed. Vigil-De Gracia conducted a study to examine deaths due to eclampsia and HELLP syndrome. They reviewed previous studies and found that from 304 maternal deaths between 1995 and 2008, 100 suffered from eclampsia, 117 suffered from HELLP and eclampsia, and 87 suffered from HELLP syndrome. Burden of eclampsia was higher in low income countries with $42.5 \%$ of deaths, while the same number was $3.9 \%$ in high income countries. Conversely, deaths due to HELLP syndrome were not dependent on the socioeconomic status of the community and had a poor outcome (12). In our study, almost half of the deaths were because of these 2 diseases. It is obvious that special preventive measures should be taken to reduce mortality $(13,14)$. Previous studies identified abdominal pain in pregnancy as an important factor (15-17), our study also reported that the most important chief complaint and the most common initial diagnosis consisted of an acute abdomen and abdominal trauma. Many studies have been conducted specifically about the management of patients with abdominal pain and many criteria have been structured to serve as a diagnostic method. Thus, it seems necessary to take actions to better tackle this issue.

\section{Conclusion}

Based on the findings it can be concluded that due to the gap between the admission and leaving the emergency department, the delay in disposition for the patients is one of the issues worth improving in order to increase maternal health. As the rate of referral in night shifts $(8$ PM to $8 \mathrm{AM}$ ) is more than day shifts, enhancing night shifts with skillful staff and necessary equipment is essential. Abdominal pain and acute abdomen have the most frequency as the initial compliant and initial diagnosis especially during the second trimester of pregnancy. In this regard, appropriate training and skills to deal with such problems are of great importance. By the same token, findings show that more than half of the pregnant women referred to the emergency department were hospitalized or admitted to ICU. Therefore, adequate facilities and space are other important matters which should be taken into consideration.

\section{Ethical issues}

This study was confirmed by the regional board of ethics at Tabriz University of Medical Sciences. Personal information of patients was kept confidential and data regarding the patients were used without their names or any means of future detection of the patient by any third party involved in the publishing of the study.

\section{Authors' contributions}

HO: study design; SSV: study conduct and data analysis; ST: supervision; ARJ: data gathering; PSM: sudy review; MM: writing and proofing.

\section{References}

1. Spence NJ. The long-term consequences of childbearing physical and psychological well-being of mothers in later life. Res Aging 2008; 30(6): 722-51. doi: $10.1177 / 0164027508322575$.

2. United Nations Secretary-General. Global strategy for women's and children's health. The Partnership for Maternal, Newborn and Child Health; 2010.

3. Say L, Chou D, Gemmill A, Tunçalp Ö, Moller AB, Daniels J, et al. Global causes of maternal death: a WHO systematic analysis. Lancet Glob Health 2014; 2(6): e323-33. doi: 10.1016/S2214-109X(14)70227-X.

4. AbouZahr C. Global burden of maternal death and disability. Br Med Bull 2003; 67(1): 1-11. doi: 10.1093/bmb/ $\operatorname{ldg} 015$.

5. Borthen I, Gilhus NE. Pregnancy complications in patients with epilepsy. Curr Opin Obstet Gynecol 2012; 24(2): 7883. doi: 10.1097/GCO.0b013e32834feb6a.

6. Nakimuli A, Nakubulwa S, Kakaire O, Osinde MO, Mbalinda SN, Kakande N, et al. The burden of maternal morbidity and mortality attributable to hypertensive disorders in pregnancy: a prospective cohort study from Uganda. BMC Pregnancy Childbirth 2016; 16: 205. doi: 10.1186/s12884-016-1001-1.

7. Moradi M. Pulmonary thromboembolism in pregnancy: Diagnostic imaging and related consideration. J Res Med Sci 2013;18(3):255-9.

8. Patel HC, Singh BB, Moitra M, Kantharia SL. Obstetric 
referrals: scenario at a primary health centre in Gujarat. National Journal of Community Medicine 2012; 3(4): 711-14.

9. Fathi Najafi T, Latifnejad Roudsari R, Hejazi M. Iron supplementation protocols for iron deficiency anemia: a comparative review of iron regimens in three countries of India, Iran and England. Journal of Midwifery and Reproductive Health 2014; 2(1): 89-96. doi: 10.22038/ jmrh.2013.2088.

10. Ndidi E, Oseremen I. Reasons given by pregnant women for late initiation of antenatal care in the niger delta, Nigeria. Ghana Med J 2010; 44(2): 47-51.

11. Solanke D, Rathi C, Pandey V, Patil M, Phadke A, Sawant P. Etiology, clinical profile, and outcome of liver disease in pregnancy with predictors of maternal mortality: a prospective study from Western India. Indian Journal of Gastroenterol 2016; 35(6): 450-8. doi: 10.1007/s12664-0160704-6

12. Vigil-De Gracia P. Maternal deaths due to eclampsia and HELLP syndrome. Int J Gynaecol Obstet 2009; 104(2): 904. doi: 10.1016/j.ijgo.2008.09.014.
13. Haram K, Svendsen E, Abildgaard U. The HELLP syndrome: clinical issues and management. A Review. BMC Pregnancy Childbirth 2009; 9: 8. doi: 10.1186/1471-2393-9-8. doi: 10.1186/1471-2393-9-8.

14. Brandenburg VM, Frank RD, Heintz B, Rath W, Bartz C. HELLP syndrome, multifactorial thrombophilia and postpartum myocardial infarction. J Perinat Med 2004; 32(2): 181-3. doi: 10.1515/JPM.2004.033.

15. Mehdizadeh A, Akbarian AR, Sarlak Z, Eghtesadi Eraghi P. Evaluation of acute abdomen in 100 pregnant women at teaching centers of iran university of medical sciences. Razi Journal of Medical Sciences 2003; 10(35): 433-9. [In Persian].

16. Kosai N, Amin-Tai H, Gendeh H, Salleh S, Reynu R, Taher $\mathrm{M}$, et al. Pregnant and severe acute abdominal pain: A surgical diagnostic dilemma. Clin Ter 2015; 166(3): 110-3. doi: 10.7417/CT.2015.1839.

17. Kennedy A. Assessment of acute abdominal pain in the pregnant patient. Semin Ultrasound CT MR 2000; 21(1): 64-77. doi: 10.1016/S0887-2171(00)90014-3. 